\title{
Towards a climatology of sensitivities of Mediterranean high impact weather - first approach
}

\author{
V. Homar ${ }^{1}$, A. Jansà ${ }^{2}$, J. Campins ${ }^{2}$, and C. Ramis ${ }^{1}$ \\ ${ }^{1}$ Meteorology Group, Departament de Física, Universitat de les Illes Balears, Spain \\ ${ }^{2}$ Centro Meteorológico en Illes Balears, Instituto Nacional de Meteorología, Spain \\ Received: 8 November 2005 - Revised: 10 February 2006 - Accepted: 14 February 2006 - Published: 10 April 2006
}

\begin{abstract}
During recent years, great interest has grown within the operational weather community on the adaptable component of observational networks. Decisions regarding where to deploy new observations of special value under threatening weather, or regarding permanent changes in observational strategies need support from sensitivity studies that determine areas where the addition of observations would optimally improve the skill of numerical predictions. Within the context of the MEDEX project (http://medex.inm. uib.es), the sensitivities of a collection of severe weather episodes in the Mediterranean have been computed using the MM5 Adjoint Modeling system. Various approaches are explored trying to summarize the results for the diversity of cases that produce high impact weather (HIW; mainly heavy rain and strong winds) in the Mediterranean region. A first attempt uses an objective classification of the trajectories of the most intense cyclone types from the ERA-40 reanalyses. Sensitivities are then computed for each group of frequent trajectories, providing a prototype sensitivity field for each of the most frequent intense cyclones in the Mediterranean. However, a large portion of HIW episodes in the Mediterranean are not linked to significantly intense cyclones within the climatology. Consequently, a subjective classification of HIW events is also performed and the sensitivity fields for an example case is shown to complete the study. Although the sensitive areas for Mediterranean HIW are not particularly confined, it is remarkable how poorly sampled areas by the regular observing networks such as North Africa and the eastern North-Atlantic are highlighted in the results.
\end{abstract}

\section{Introduction}

Operational weather prediction centers are receiving increasing pressure from the public and the authorities to extend and improve the forecasts while reducing costs and increas-

Correspondence to: V. Homar

(victor.homar@uib.es) ing efficiency. The European community is strongly committed to accomplish this increased efficiency on the observational component of the weather forecasting process. The Network of European Meteorological Services Composite Observing System project (EUMETNET-EUCOS, http: //www.eumetnet.eu.org/conteucos.html) is appointed to optimize the composite observing system at European scale with a view to improve short range forecast over Europe without increasing significantly the overall cost. EUCOS recognizes the necessity to identify data sparse areas around Europe, and more specifically, the first objective of EUCOS high level design is to clearly identify the areas where an increased observation effort needs to be implemented in order to improve numerical weather predictions. To this end, the EUCOS programme established as a priority the generation of a climatology of forecast sensitivities. At European scale, in average, sensitivities of forecast errors over Europe are located mainly upstream of the westerlies, over the Northeastern Atlantic (Marseille and Bouttier, 2000). However special interest exists in analysing the sensitivities of high impact weather (HIW) due to the larger associated benefits of potential forecast improvements. It is well known that the Mediterranean region is persistently affected by hazardous weather such as strong windstorms or heavy rains that produce extreme floods.

The Mediterranean experiment on cyclones that produce high impact weather in the Mediterranean (MEDEX, http://medex.inm.uib.es) is a project endorsed by theWorld Weather Research Program of the World Meteorological Organization that coordinates weather services and research institutions from most countries of the Mediterranean basin. MEDEX is designed to contribute to the basic understanding and short-range forecasting of HIW events in the Mediterranean, mainly heavy rain and strong winds. One milestone within this project is the calculation of sensitive areas where an increase in the number, quality or type of observation would improve the numerical prediction of HIW episodes. 


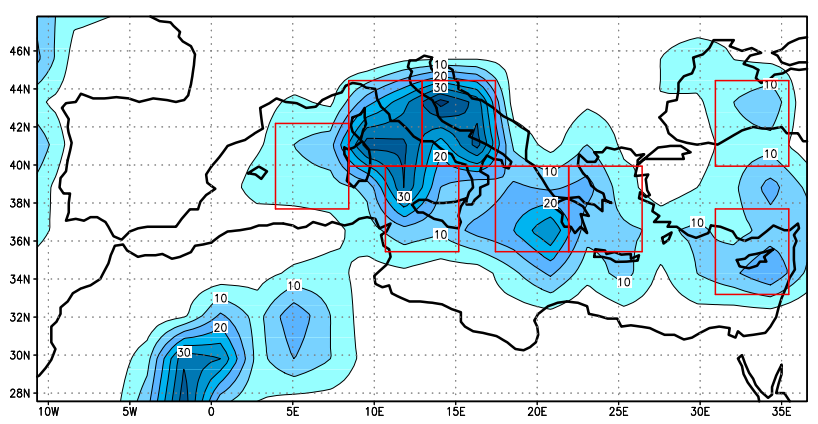

$\begin{array}{llllllll}5 & 10 & 15 & 20 & 25 & 30 & 35 & 40\end{array}$

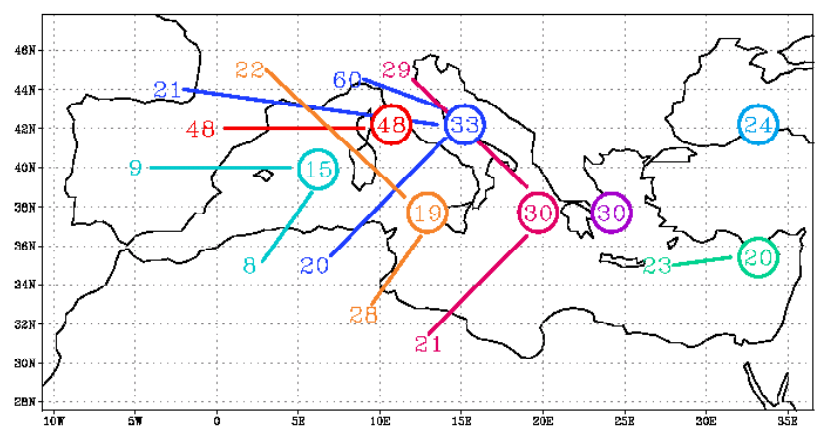

Fig. 1. Upper panel) Number of intense cyclones that achieve maximum circulation within the $2.25^{\circ} \times 2.25^{\circ}$ gridbox over the 45 years ERA-40 period. Lower panel) Classification of each type of intense cyclones as defined in the upper panel with number of members in each class (see Table 1). The numbers in circles correspond to cyclones that achieve the maximum circulation within the genesis area and numbers connected to the region by a line indicate the number of cyclones reaching the area from that direction.

Provided the common interest between EUCOS and MEDEX, collaboration and support between the two projects were established, and an agreement was formalized to build a climatology of short-range sensitive areas for HIW events in the Mediterranean. This paper presents the first steps towards the construction of the climatology of HIW sensitivities, with a discussion about the obstacles and difficulties found.

\section{Methodology}

In order to build the climatology of short-range sensitivities of high impact weather in the Mediterranean, a welldefined climatology of such events consistent across the region should be used as a starting point. As far as we know, the most recent effort to build such a climatology is being done within the MEDEX community. However, many difficulties have arisen during the data collection process. The climatology of Mediterranean HIW is currently a list of cases selected by the project members as important and representative of HIW in their region (http://medex.inm.uib.es/data/ Selection_cases.htm). This list is not exhaustive and does not include systematic information, neither meteorological nor about the societal impacts, for all events. Thus, since a HIW database is not currently readily available, alternatives to systematize the identification of HIW are explored.

A natural approach is to use the strong link between HIW and intense cyclones. Although most of HIW events are linked to cyclones (Jansà et al., 2001), not all of these cyclones are intense. However, intense cyclones are naturally associatted with HIW. In an attempt to identify most of the events that produce high impact in the Mediterranean, while admitting the inadequacy of the available HIW databases, we use intense cyclones as a proxy for HIW events. Indeed, a cyclones database has been developed under the MEDEX framework (publicly available at http://medex.inm.uib.es) and it is used here to identify characteristic intense cyclones that produce HIW across the Mediterranean. The cyclones catalogue is based on the Reanalysis fields from the European Center for Medium-Range Weather Forecast (ERA-40) and characterizes the size, position, intensity, depth and path of all sea-level pressure cyclones within a domain that covers the Mediterranean region (see Fig. 1). The detection and tracking algorithms as well as the characterization methods are described in detail in Picornell et al. (2001) and Campins et al. (2006).

Intense cyclones in this study are selected as those cyclonic systems with a maximum circulation exceeding $7 \times 10^{7} \mathrm{~m}^{2} \mathrm{~s}^{-1}$ and a lifetime of at least $24 \mathrm{~h}$. The intensity is computed as an area integral of the geostrophic vorticity at $1000 \mathrm{hPa}$ over the area around the cyclone's center with positive geostrophic vorticity.Over the 45 years period covered by the ERA-40 data (September 1957 to August 2002), 1359 intense cyclones were identified in the study domain which represents a mean frequency exceeding 30 intense cyclones per year. The upper panel in Fig. 1 clearly depicts the areas where the most intense cyclones are frequently detected in the Mediterranean. In an attempt to regionalize the intense cyclones and later compute sensitivities of the associated HIW events, we divide the distribution shown in Fig. 1 into eight regions. Note that unlike the cyclones detected over the sea, the two maxima obtained over north Africa mostly represent large thermal lows that fit the criteria of circulation due to size but do not have high vorticity or strong winds associated with them. These cases will be ignored in this study. The eight regions, and the frequency of cyclones in each one are labeled in Table 1. In order to consider some kind of physical mechanisms leading to the cyclone intensification within the boxes, the path of all cyclones for each region is analysed and a classification depending on the origin of the cyclone is performed. Bottom panel of Fig. 1 shows the number of detected cyclones for the most frequent classes for each of the eight regions.

Each of these classes correspond to a type of intense cyclone that we use as a proxy for HIW in the Mediterranean basin. At this point of the study, we compute the sensitivity fields for each class applying the analysis on a single episode belonging to the class. Although the criteria used to define the classes (common area of maximum circulation and common origin) do not guarantee homogeneity of sensitivities across the class members, and thus the representativity of 
Table 1. Regions and classes of intense cyclones classified from the 45 years of ERA-40 analysis. The origin of the cyclones in the class is indicated by $\mathrm{N}, \mathrm{W}$ and $\mathrm{S}$. Classes 0 indicate cyclones with its origin over the same region where they achieve maximum circulation. The numbers next to the region name represent the total number of cyclones achieving its maximum circulation in that region. The numbers in parenthesis show the percentile frequency of that class within the region and with respect to all intense Mediterranean cyclones. The total number of intense cyclones detected for the 45 years period is 1359 .

\begin{tabular}{|c|c|c|c|}
\hline Region & Class (\%) & Region & Class (\%) \\
\hline \multirow{5}{*}{$\begin{array}{c}\text { Adriatic (Ad) } \\
134\end{array}$} & $0(25,2.4)$ & \multirow{3}{*}{ Sicily (Si) 74} & $0(26,1.4)$ \\
\hline & $\mathrm{N}(45,4.4)$ & & $\bar{N}(30,1.6)$ \\
\hline & $\mathrm{W}(16,1.5)$ & & $\mathrm{S}(38,2.1)$ \\
\hline & \multirow{2}{*}{$\mathrm{S}(15,1.5)$} & \multirow{2}{*}{ Cyprus (Cy) 54} & $0(37,1.5)$ \\
\hline & & & $\mathrm{W}(43,1.7)$ \\
\hline \multirow{2}{*}{$\begin{array}{l}\text { Tyrrhenian (Ty) } \\
120\end{array}$} & $0(40,3.5)$ & Aegean $(\mathrm{Ae}) 47$ & $0(64,2.2)$ \\
\hline & $\bar{W}(40,3.5)$ & Black Sea (BS) 40 & $0(60,1.8)$ \\
\hline \multirow{3}{*}{$\begin{array}{c}\text { Ionian (Io) } \\
80\end{array}$} & $0(38,2.2)$ & \multirow{3}{*}{ Balearic (Ba) 39} & $0(38,1.1)$ \\
\hline & $\mathrm{N}(36,2.1)$ & & $\overline{\mathrm{W}}(23,0.7)$ \\
\hline & $\mathrm{S}(26,1.5)$ & & $\mathrm{S}(21,0.6)$ \\
\hline
\end{tabular}

the results may not be significant for all classes, the sample of sensitivity fields presented here already shows illustrative features.

For each class a single case is selected and the sensitivity fields are computed using the MM5 Adjoint modeling system (Zou et al., 1997, 1998). The adjoint model $(L)$ is a tangent linear operator that traces the gradients vector of a response function $\left(\nabla_{x} J\right)$ with respect to the forward (standard nonlinear) model fields $(\boldsymbol{x})$ back in time, along forward model trajectories in phase space (see Errico, 1997, for an introduction to adjoint techniques in numerical weather prediction). As a result, the gradients of the response function, defined at a forecast time, with respect to the model fields at an earlier time (usually the initial conditions time) are obtained:

$$
\left.\nabla_{x} J\right|_{t=t_{0}}=\left.L \nabla_{x} J\right|_{t=t_{f}}
$$

Where $t_{0}$ is the initial conditions time, $t_{f}$ the forecast time when the response function $(\mathrm{J})$ is defined. These fields inform about the changes in the forecast that will be obtained for a certain perturbation to the model fields, which is commonly referred to as the sensitivity of the forecast to the model fields. The selection of $J$ is a key point in the construction of the climatology of sensitivities. Any sensible comparison between different sensitivity fields must be done using the same response function or a balance relation that links them. Since all episodes under this part of the study are connected to an intense cyclone, we define as response function the $48 \mathrm{~h}$ forecast pressure field at the 3 lowest $\sigma$ model levels over an area of $240 \times 240 \mathrm{~km}^{2}$ centered on the predicted cyclone minimum pressure. Note that the location of the predicted cyclone center may differ from the location of the cyclone in the classifying database, thus bringing parts of the response function off the limits of the areas depicted in Fig. 1. Although humidity involves highly nonlinear processes that hamper the applicability of the adjoint model (Vukićević and

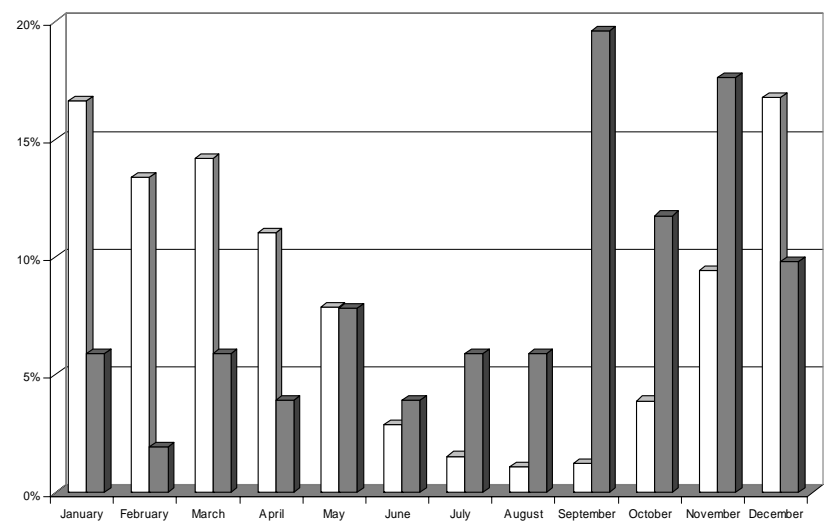

Fig. 2. Monthly frequency of intense cyclones in the ERA-40 analysis 1957-2002 (white) and monthly distribution of episodes in the MEDEX list of selected cases (grey).

Errico, 1993), we include explicit moisture terms in the adjoint model run for being most likely an influential factor in many Mediterranean HIW events that must be considered in the sensitivity calculations. For a more detailed description of the adjoint model and its application to a Mediterranean cyclone, see Homar and Stensrud (2004). The adjoint model computes the gradients of the response function with respect to each model field on the initial conditions. A way of summarizing the results is to compute the vertical average of all sensitivities, which provides a perception of the most sensitive regions, ignoring all information about the sensitivity of the response function to different levels or fields and resulting in a field with non-physical units ([Pressure units]/["IC units"], Homar and Stensrud, 2004). This vertical averaging is the standard product, hereafter referred to as sensitivity, used in this study to summarise the complete set of gradients for each case and help the comparison among different regions and classes.

Using a catalog of intense cyclones as a proxy seems like a sensible way of systematizing the starting point of the climatology of sensitivities when no climatology of Mediterranean HIW per se is available. However, when comparing the annual distribution of intense cyclones and episodes of HIW, it is clear that this approach is missing many important events that occur in late summer and autumn (Fig. 2). While most of intense cyclones are detected during winter, most of the cases reported to the MEDEX list occurred during late warm season. Consequently, an effort to classify the episodes available in the MEDEX list of selected cases has been done. Since no completeness of the list is guaranteed and the data available for each case is not homogeneous, the classification is necessarily subjective. Analysing each case individually, we divide all episodes in 4 classes depending on the regional origin of the feature responsible of the HIW: Genoa, Cyprus, Algerian-Tunisian coast and East Iberia-Gulf of Lyon. The first two classes are episodes which are typically associated with intense cyclones and so are well represented by the previous approach. On the other hand, 


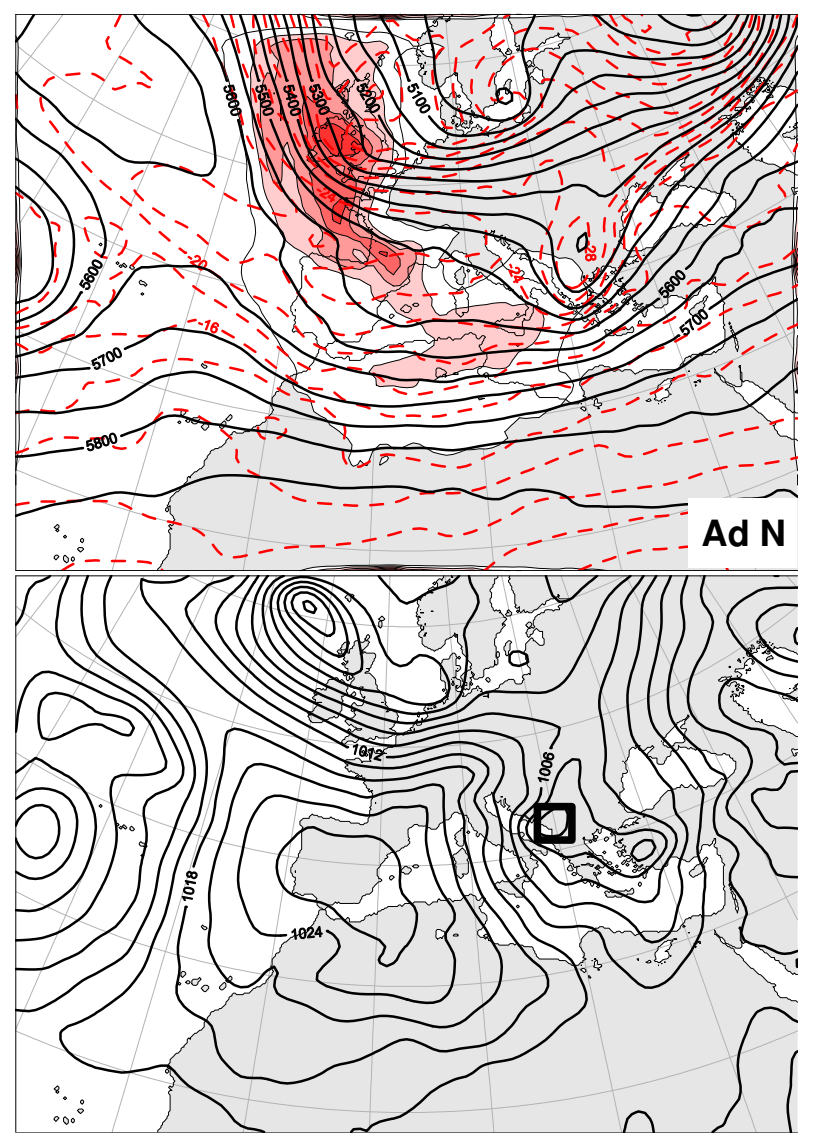

Fig. 3. Upper panel) Geopotential height ( $\mathrm{m}$, solid) and temperature $\left({ }^{\circ} \mathrm{C}\right.$, dashed) at $500 \mathrm{hPa}$ for a case of the Adriatic reaching the region from the north (fields valid on 25 December 2001 at 12:00 UTC). Shading shows the vertically averaged adjoint-computed sensitivity fields (darker colors show larger sensitivities). Lower panel: Sea-level pressure forecast by standard MM5 valid on 27 December 2001 at 12:00 UTC. The area used to define the response function and initialize the adjoint model is indicated with a square

most cases in the Algerian-Tunisian coast and East IberiaGulf of Lyon are connected to moderate or weak perturbations. These episodes need to be explored individually and summarizing results becomes a difficult task owing to the heterogeneity of mechanisms involved in different episodes. An example of this type of events is presented in Sect. 3.2.

\section{First sensitivity calculations}

\subsection{Intense cyclone events}

Figures 3-10 show the sensitivities for single cases belonging to 8 frequent classes of intense cyclones in the Mediterranean. Note that the results for all regions and classes listed in Table 1 are not shown here. The complete set of results will be made publicly available through the MEDEX web page (http://medex.inm.uib.es). All figures show the location of the response function on the $48 \mathrm{~h}$ forecast sea-level

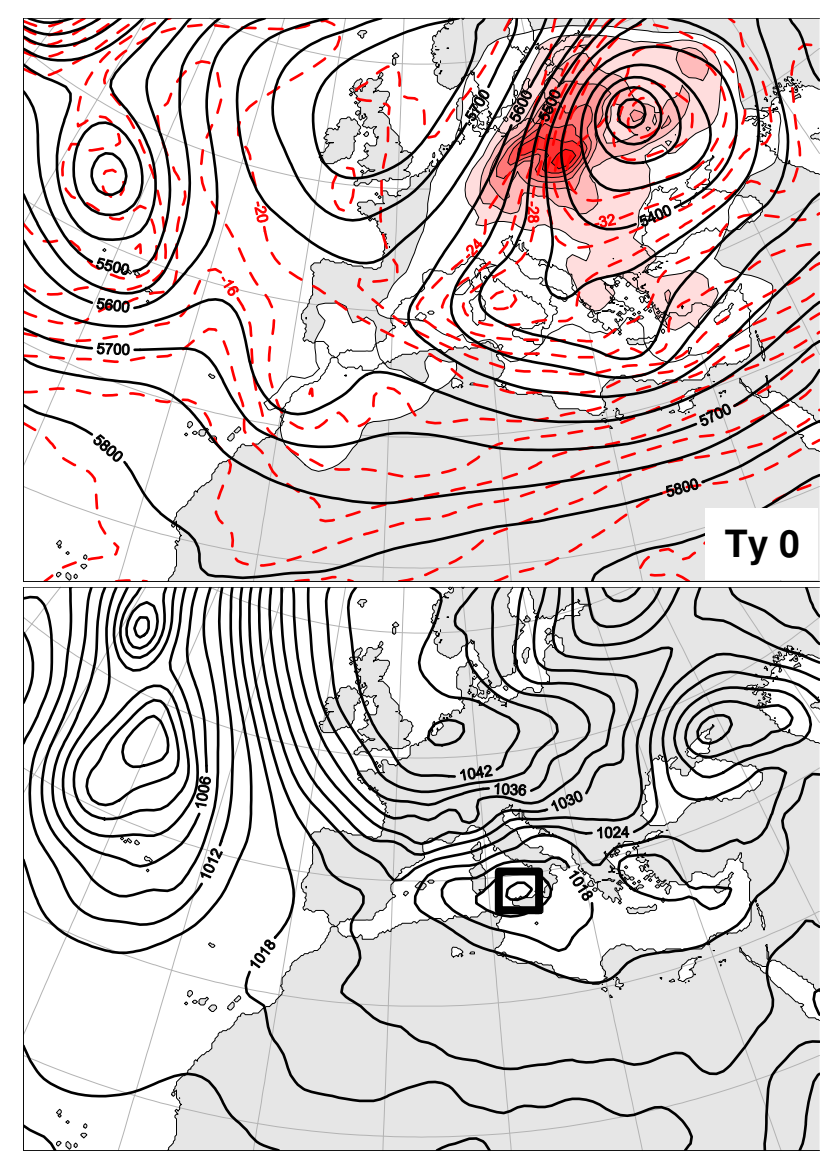

Fig. 4. Same as in Fig. 3 for a case of the Tyrrhenian generated within the same area. Upper panel fields valid on 12 December 2001 at 06:00 UTC and lower panel field valid on 14 December 2001 at 06:00 UTC.

pressure field and the resulting sensitivity field on top of the geopotential and temperature initial conditions fields at $500 \mathrm{hPa}$.

For the sake of brevity, only general notable aspects of the sensitivity fields will be discussed. Details for each class are likely particular of the selected case instead of representative of the group and will not be described. A first common feature among all classes is the high sensitivity values obtained over areas with large horizontal gradients at mid-tropospheric fields on the precursor system. This common aspect indicates the tipically baroclinic character of the cyclogenesis conducive to intense cyclones in the Mediterranean, highlighting the mid-upper tropospheric precursor trough as the main contributor to the low-level cyclogenetic forcing and thus as determinant for the forecast details of the intense cyclone (Reiter, 1975). The distance from the region of high sensitivities, where special observations would be deployed in a hypothetical targeting campaign, to the potentially hazardous weather region varies largely from case to case. The differences between $A e O$ and $C y W$ (Figs. 7 and 9 ) are illustrative. The $C y W$ cyclone is well formed at the beginning of the forecast period and remains quasi-stationary 


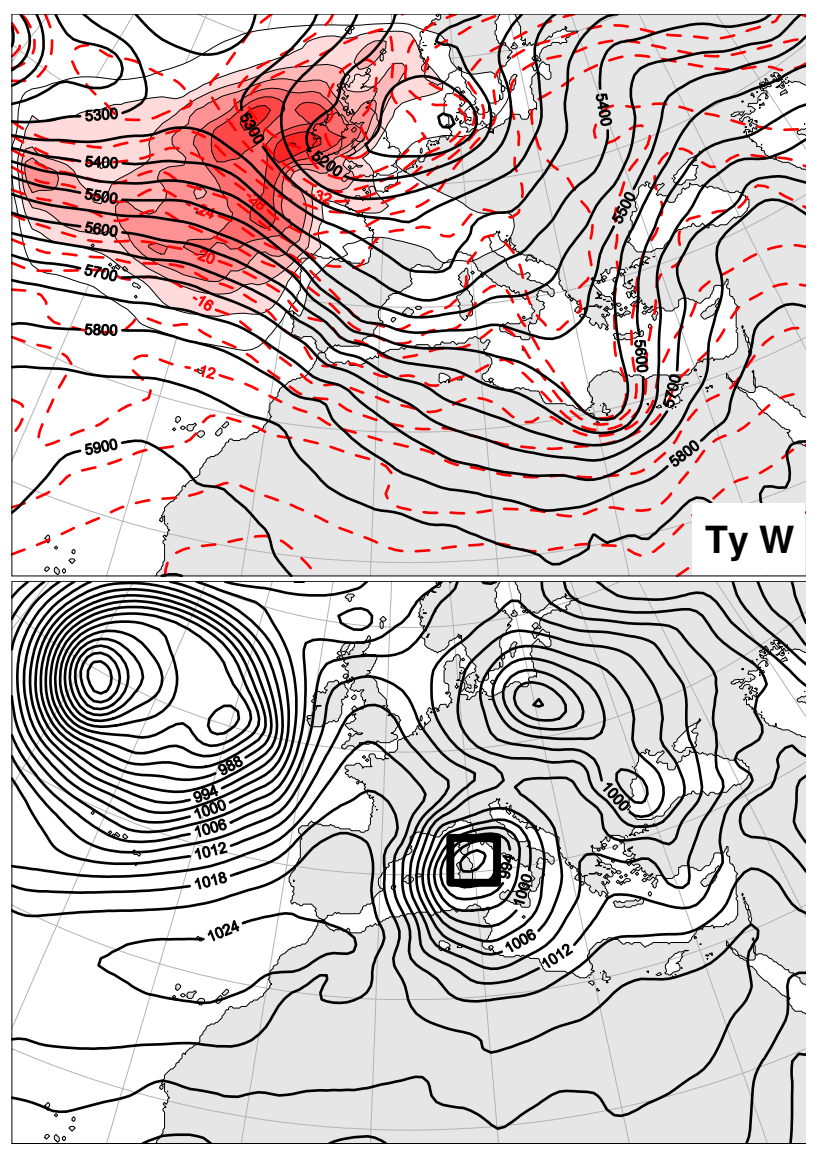

Fig. 5. Same as in Fig. 3 for a case of the Tyrrhenian reaching the area from the West. Upper panel fields valid on 29 December 2000 at 00:00 UTC and lower panel field valid on 31 December 2000 at 00:00 UTC.

during the $48 \mathrm{~h}$ time span whereas the Ae 0 episode shows a fast evolution of the mid and upper-level system that leads to the Aegean intense cyclone $48 \mathrm{~h}$ in the forecast. As a result, the $48 \mathrm{~h}$ sensitivities for the $A e O$ are located far west, well over the North Atlantic and the sensitive areas for the $C y W$ remain in the neighbouring region, partially over north Africa.

Regarding the location of the highest values of sensitivity, this small sample of classes already show large dispersion. The Ad N, Ty W, Ad O, Io O, Ae O,Cy W and Ba S classes show high values of sensitivity over poorly sampled water bodies as the North Atlantic and the Mediterranean. On the other hand, most of the sensitive areas obtained for classes $T y O$ and Io 0 are routinely adequately sampled by operational surface and upper-air in situ observational networks. Concerning the north-African lands, the $I o O, C y W$ and $B a S$ episodes mainly, and $A d N$ with minor intensity, show sensitivity of the $48 \mathrm{~h}$ forecast over North-Africa.

In a first attempt to summarize the abundant information from the various regions and classes defined in this study, and taking advantage from the fact that a common response function is used for all experiments, a mean weighted sensi-

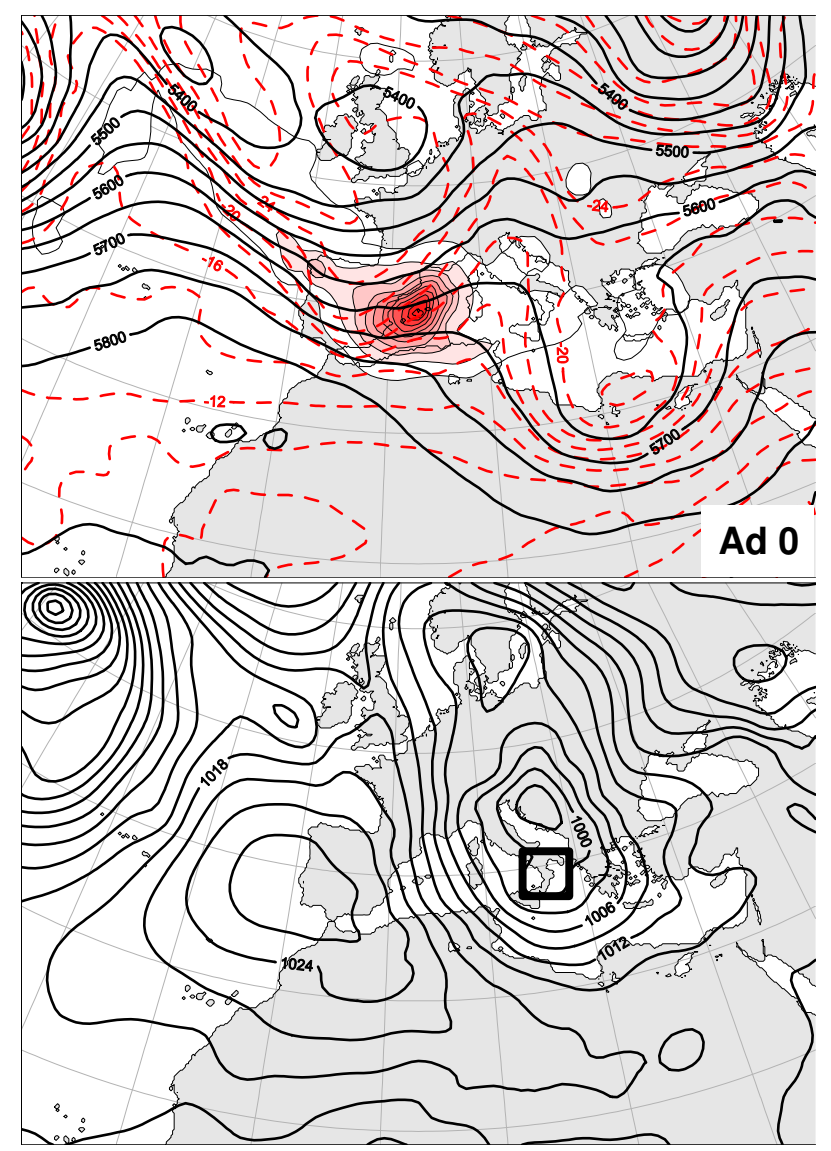

Fig. 6. Same as in Fig. 3 for a case of the Adriatic generated within the same area. Upper panel fields valid on 29 November 1997 at 00:00 UTC and lower panel field valid on 1 December 1997 at 00:00 UTC.

tivity field of intense cyclones in the Mediterranean is produced. Each sensitivity field is weighted by the frequency of the intense cyclone class it represents (Table 1). It is mandatory to bear in mind that this is just an experimental product that does not include at this stage of the research all classes in Table 1 but just the most frequent types of intense cyclones shown in Figs. 3-10. The resulting mean field is shown as an example of the kind of products that can be obtained from a climatology of sensitivities, which can guide decision-makers in defining new observational strategies in an informed cost/loss basis. Figure 11 depicts this field, highlighting four main regions of $48 \mathrm{~h}$-sensitivity of intense cyclones forecasts within the reference domain: east North Atlantic, central and eastern Europe, Eastern Mediterranean and Western Mediterranean with north African lands. Small scale features in Fig. 11 are just an artifact of the reduced sample size used to compute the weighted mean, and thus irrelevant. Focusing on areas with poor in situ measurements, this result highlights primarily the east North Atlantic as the area where upper-air disturbances penetrate the continent and are eventually conducive to intense cyclones that produce HIW in the Mediterranean region. Also the Mediter- 


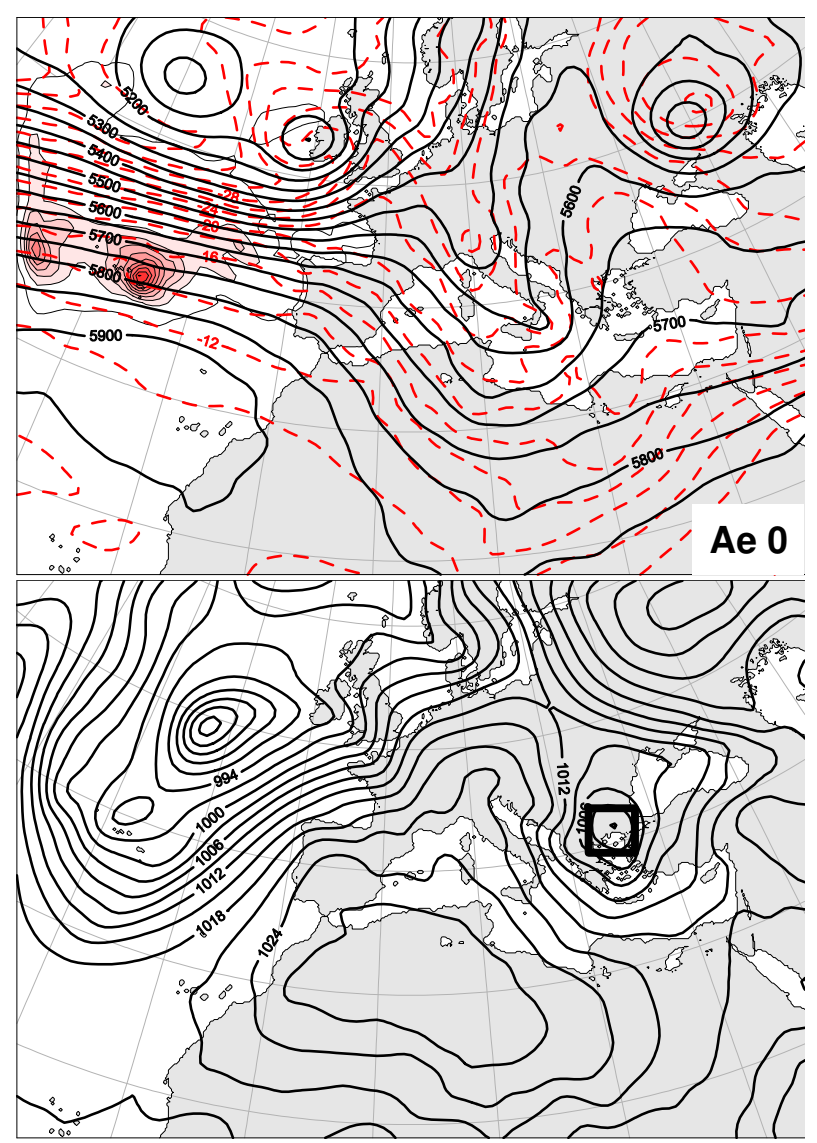

Fig. 7. Same as in Fig. 3 for a case of the Aegean generated within the same area. Upper panel fields valid on 25 November 2000 at 12:00 UTC and lower panel field valid on 27 November 2000 at 00:00 UTC.

ranean sea and north Africa are emphasized by the mean sensitivity field. It is noteworthy that within the scope of the EUMETNET-EUCOS project, contrasting with the North Atlantic region, the North of Africa and the Mediterranean basin are areas of sensitivity exclusively for Mediterranean European countries. The areas important for the forecast of HIW in Central and North Europe do not likely reach the Mediterranean and North Africa being primarily concentrated upstream of the storm track, over the North Atlantic.

Besides the geographical distribution of the sensitivity fields, a measure of the amount of sensitivity shown by a response function gives a measure of the predictability of the episode with this numerical configuration. Under the hypothesis that the sensitivities derived from the adjoint are reliable, the larger the sensitivity values, the lower the predictability of the response function since small perturbations to the initial conditions will produce larger variations in the forecast field. Again, since the same response function is used for all experiments, the resulting fields are consistent and the comparison among them is relevant. The root mean square of the vertically averaged sensitivity field is chosen as a gross measure of the magnitude of the sensitivity shown by each

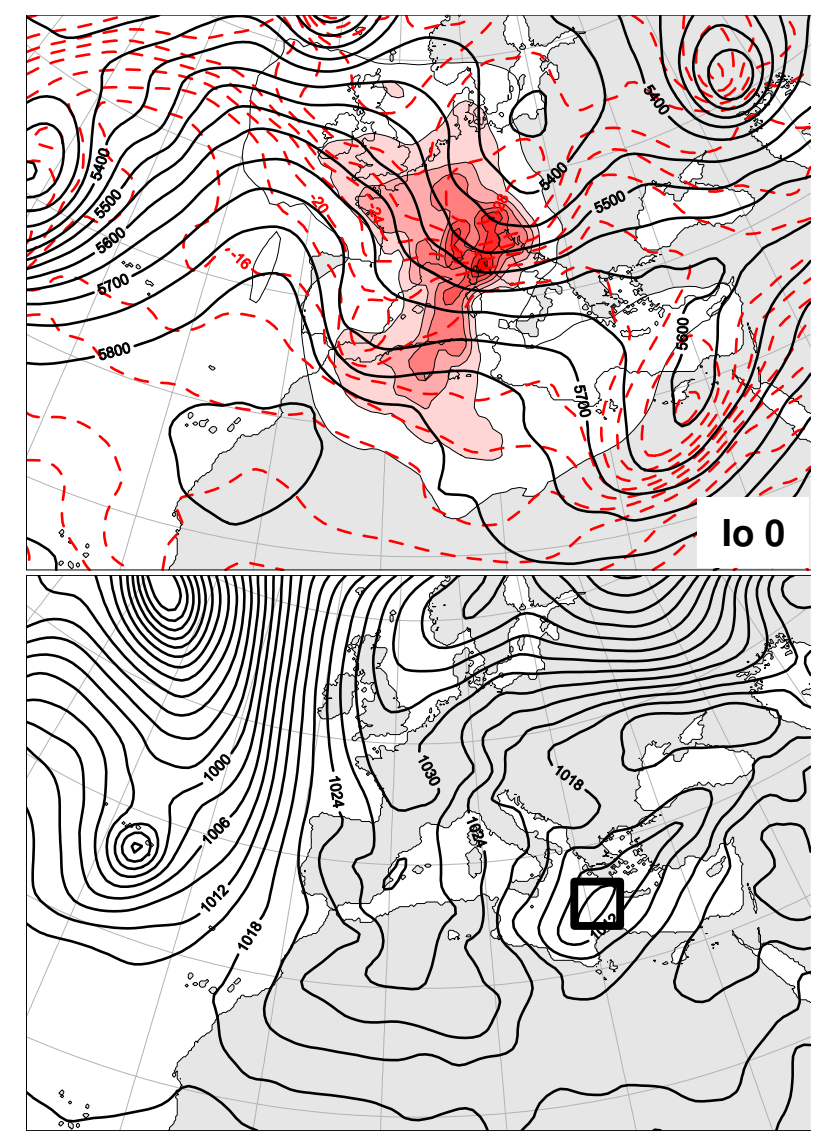

Fig. 8. Same as in Fig. 3 for a case of the Ionian generated within the same area. Upper panel fields valid on 12 December 1997 at 18:00 UTC and lower panel field valid on 14 December 1997 at 18:00 UTC.

episode (Fig. 12). The episode representative of the Ae 0 class shows very high values which can be interpreted as the very limited predictability of the sea level pressure $48 \mathrm{~h}$ in the forecast over the box defining the response function (Fig. 7, lower panel) under a fast progressing upper-level trough. Indeed, slight changes in the shape and intensity of the gradients of the mass and wind fields during the progression of the system will vary the wave velocity and thus change dramatically the forecast (and so the response function). On the other hand, the $C y W$ class show much higher predictability under this forecast framework due to the stationary conditions of the deep cyclone during the standard $48 \mathrm{~h}$ forecast time span. Thus, the model forecast will not be dramatically altered under small perturbations to the initial conditions. Results such as those obtained for the $A d N$ class are more difficult to interpret, because it has many similarities with the $A e O$ results, although the orography might have played a more determinant role on the cyclogenesis and intensification of the cyclone, thus favouring the stability of the forecast (i.e., increasing the predictability). 

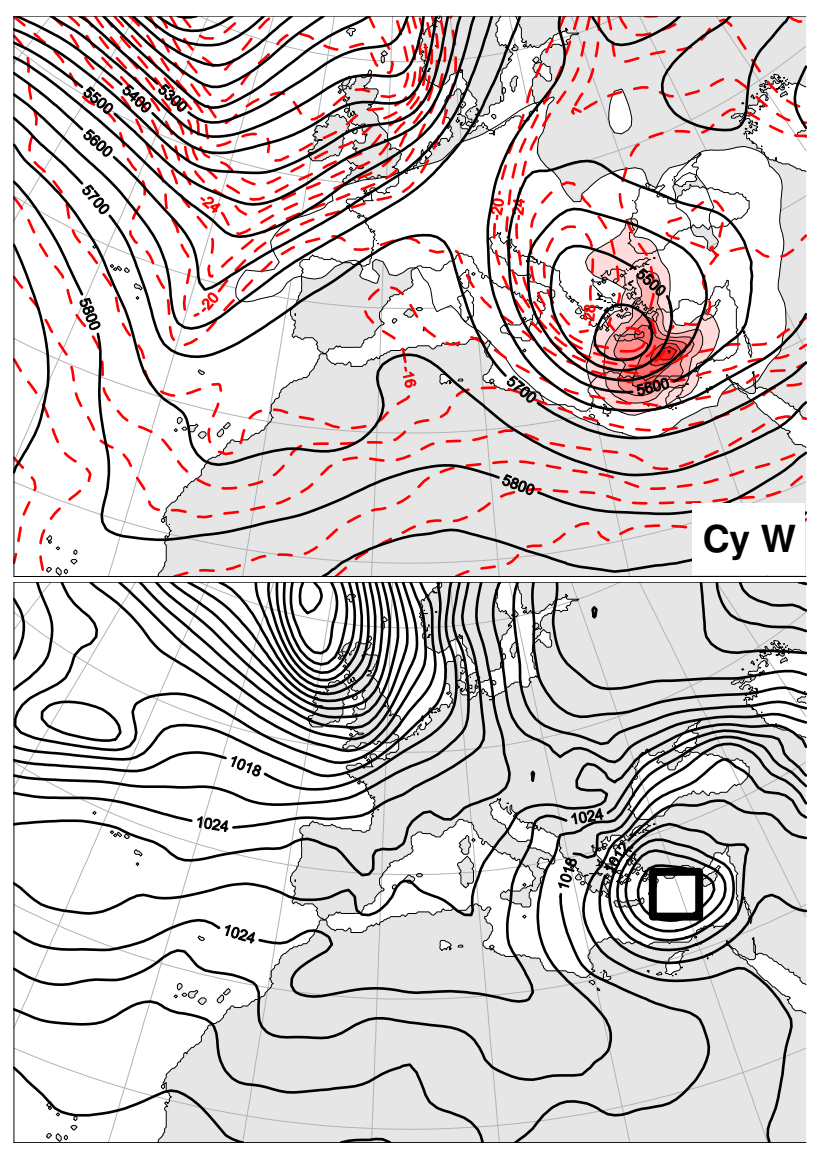

Fig. 9. Same as in Fig. 3 for a case of the Cyprus region reaching the area from the West. Upper panel fields valid on 2 December 2001 at 00:00 UTC and lower panel field valid on 4 December 2001 at 00:00 UTC.

\subsection{Non-Intense cyclone events}

In Sect. 2 we discussed the difficulties that hamper the generation of an exhaustive climatology of sensitivities of HIW in the Mediterranean. The cases not connected to an intense cyclone are not considered at all in the approach presented in the previous section. Since no systematic climatology of HIW is available, the variety of HIW impede the task of homogenizing the sensitivity calculations and so a case by case analysis needs to be done. A clear example of the difficulty is the selection of a common response function. While in the previous section the response function was selected according to the classification process (i.e. the presence of an intense cyclone in the forecast was guaranteed), a much larger diversity of possible dynamical features linked to the HIW are found in the MEDEX list of selected cases.

An example of one case with no intense cyclone associated with it is presented in Fig. 13. For this episode, $24 \mathrm{~h}$-accumulated precipitation exceeding $200 \mathrm{~mm}$ were registered in parts of Catalonia (eastern Spain) and instead of a deep mature cyclone, a low-level jet impinging towards the region is forecast by the model. Accordingly, we define the
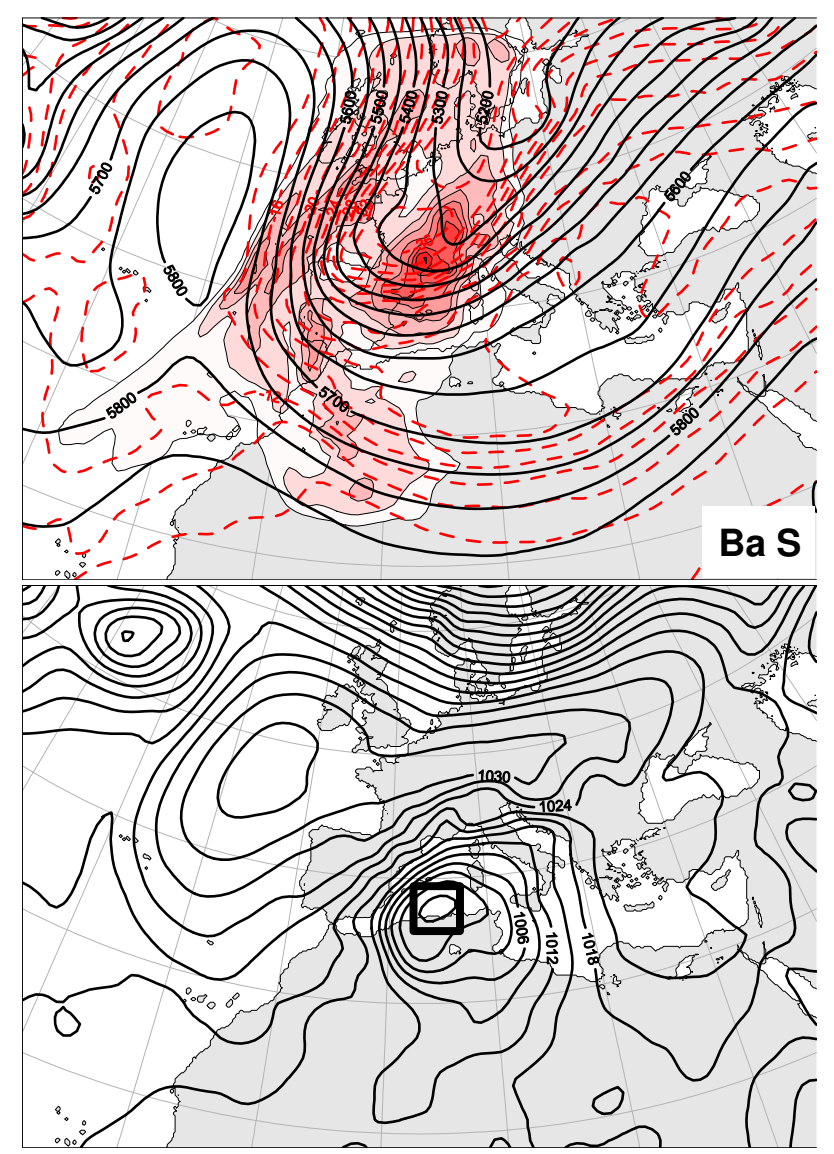

Fig. 10. Same as in Fig. 3 for a case of the Balearic region reaching the area from the South. Upper panel fields valid on 9 November 2001 at 00:00 UTC and lower panel field valid on 11 November 2001 at 00:00 UTC.

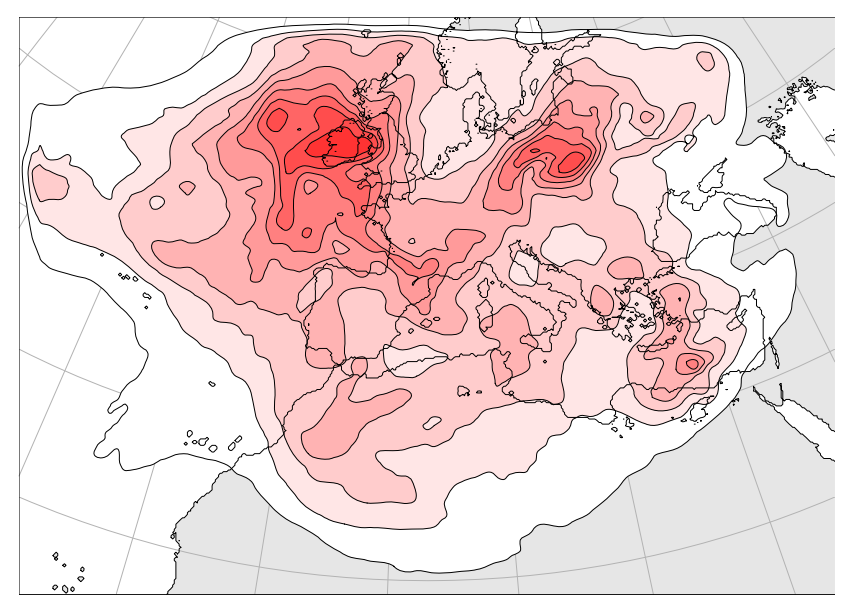

Fig. 11. Weighted mean sensitivity for frequent intense cyclones in the Mediterranean (darker color show larger mean sensitivity). Each class is weighted with its relative frequency with respect to the total intense cyclones. 


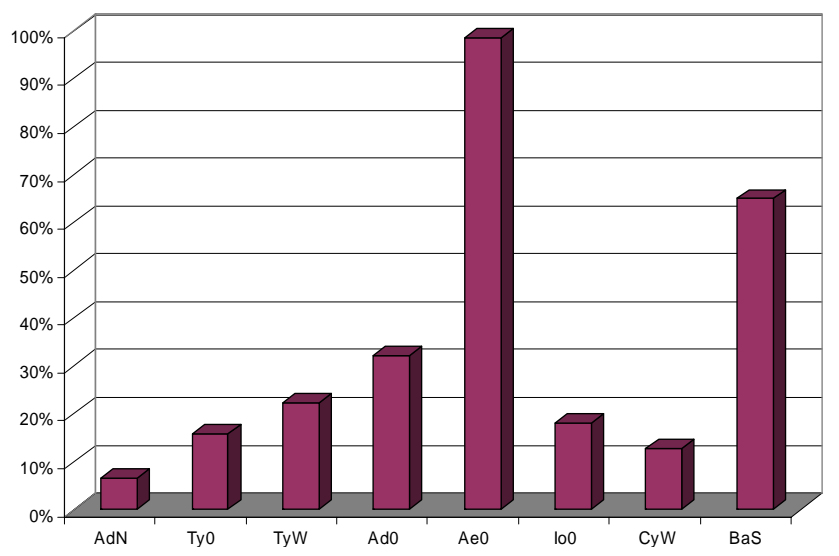

Fig. 12. Root mean square of the sensitivity field relative to a reference value for the intense cyclone classes discussed in the text.

response function for this episode as the $v$ component of the wind over the area highlighted in the lower panel of Fig. 13. The sensitivity field is also computed with the adjoint model, and show similar features to the sensitivities presented in the previous section, but no further calculations among multiple cases can be done owing to the heterogeneity of the response functions needed to adequately represent the features influential on the generation of HIW for each particular episode.

\section{Conclusions}

This paper presents the first results of an attempt to build a climatology of sensitivities of high impact weather in the Mediterranean. The research is framed within an agreement between the EUMETNET-EUCOS and MEDEX projects that identified a common interest in determining areas where an increased effort in improving observations would most efficiently improve the forecast of severe weather in the Mediterranean.

Due to the lack of a consistent climatology of HIW episodes with uniform and systematically collected data, we explore different approaches to build the climatology of sensitivities. A first test takes into account the connection between Mediterranean HIW and cyclones, and makes use of a catalogue of cyclones readily available from the MEDEX project. We use the most intense cyclones in the database as a proxy for HIW. The subset of intense cyclones are classified by regions and directions of origin. Then, the adjoint model is used to compute sensitivities for each class, resulting in a collection of sensitivity fields representative of prototype HIW in the Mediterranean. The preliminary results highlight typical sparse data collection areas such as the north Atlantic, the Mediterranean basin and north Africa as determinant for the forecast of HIW in the Mediterranean. Areas that are regularly well sampled, such as the European continent, are also highlighted but significant improvements derived from additional observational efforts over these areas are very unlikely. It is well known that the North Atlantic is important for the general weather forecast over Europe, if not for anything else

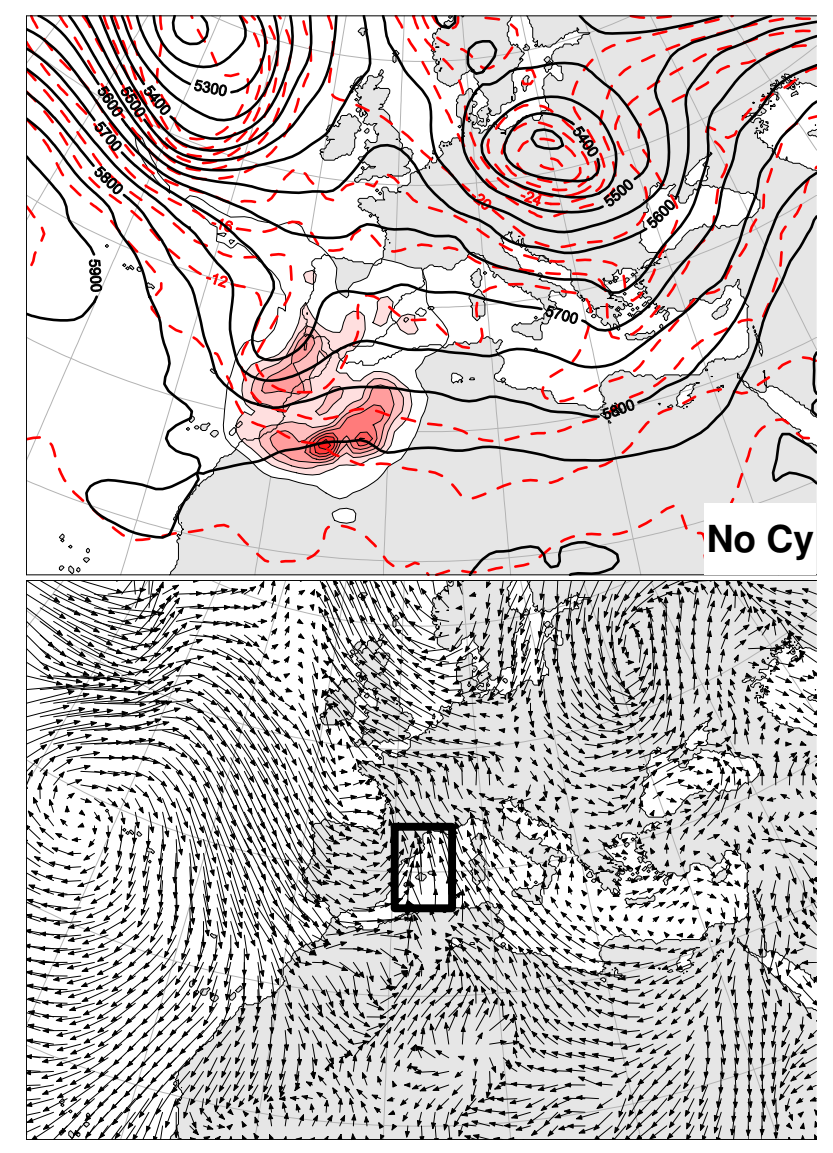

Fig. 13. Upper panel) Geopotential height (m, solid) and temperature $\left({ }^{\circ} \mathrm{C}\right.$, dashed) at $500 \mathrm{hPa}$ for a HIW case with not intense cyclone nearby (fields valid on 8 October 2002 at 00:00 UTC). Shading shows the vertically averaged adjoint-computed sensitivity fields. Lower panel: Wind field at $1000 \mathrm{hPa}$ forecast by the standard MM5, valid on 9 October 2002 at 03:00 UTC. The area used to define the response function and initialize the adjoint model is indicated with a square.

just for being upstream in the synoptic storm track. Thus, this area is likely going to be a focus point in future plans for modification of the current in situ observational strategies. However, should these results be confirmed by the ongoing research, special attention must be put on the Mediterranean basin and the north African countries in order to systematically improve the forecasts of high impact weather in the Mediterranean.

In the future, we expect more significant results by making the intense cyclones classification in a more objective way. For instance, the use of clustering techniques may produce groups of episodes with similar evolution of the synoptic pattern, which would lead to more class-representative sensitivity fields. The consistency of results within a cyclone class should also be verified. Regarding the episodes not linked to an intense cyclone, some progress could be made by finding a common response function that allows to quantitatively compare and perform inter-episode calculations with the resulting sensitivity fields. 
Acknowledgements. The authors aknowledge the ECMWF for the ERA-40 reanalysis, and thank the Mediterranean Studies Section of the Centro Meteorológico en Illes Balears for building the cyclone database. The computational resources allocated by the Instituto Nacional de Meteorologia are also aknowledged. This work has been partially funded by EUCOS, CICYT REN2002-03482 and CICYT CGL2005-05681 grants.

Edited by: V. Kotroni and K. Lagouvardos

Reviewed by: N. Prezerakos

\section{References}

Campins, J., Jansà, A., and Genovés, A.: Three-dimensional structure of Western Mediterranean cyclones, Int. J. Clim., in press, 2006.

Errico, R. M.: What is an Adjoint Model?, Bull. Amer. Meteor. Soc., 78, 2577-2591, 1997.

Homar, V. and Stensrud, D. J.: Sensitivities of an intense Mediterranean cyclone: analysis and validation, Q. J. R. Meteorol. Soc., 130, 2519-2540, 2004.
Jansà, A., Genovés, A., Picornell, M. A., Campins, J., Riosalido, R., and Carretero, O.: Western Mediterranean cyclones and heavy rain, Meteorol. Appl., 8, 43-56, 2001.

Marseille, G. J. and Bouttier, F.: Climatology of sensitive areas, ECMWF study for EUCOS, Tech. Rep. EUCOS Rep-30., 2000.

Picornell, M. A., Jansà, A., Genovés, A., and Campins, J.: Automated database of mesocyclones from HIRLAM(INM) $-0.5^{\circ}$ analyses in the Western Mediterranean, Int. J. Clim., 21, 335354, 2001.

Reiter, E. R.: Handbook for forecasters in the Mediterranean, Naval Postgraduate School, Monterey, California, 1975.

Vukićević, T. and Errico, R. M.: Linearization and adjoint of parameterized moist diabatic processes, Tellus, 45A, 493-510, 1993.

Zou, X., Vandenberghe, F., Pondeca, M., and Kuo, Y.-H.: Introduction to adjoint techniques and the MM5 adjoint modeling system, NCAR Tech. Note NCAR/TN-435+IA, 1997.

Zou, X., Huang, W., and Xiao, Q.: A User's Guide to the MM5 Adjoint Modeling System, NCAR Tech. Note NCAR/TN-437+IA, 1998. 